
\title{
Research Sulure \\ Feasibility of a Patient-Reported Outcomes Quality of Life Instrument to Improve Care in Underserved, Ethnic Minority Cancer Patients
}

Nandita Khera ( $\nabla$ khera.nandita@mayo.edu )

Mayo Clinic Arizona

lan Komenaka

Maricopa Integrated Health System

Gilbert Ramos

Maricopa Integrated Health System

Briant Fruth

Mayo Clinic Minnesota

Waqas Arslan

Maricopa Integrated Health System

Donald Northfelt

Mayo Clinic Arizona

Rahma Warsame

Mayo Clinic Minnesota

Jeff Sloan

Mayo Clinic Minnesota

Joan Griffin

Mayo Clinic Minnesota

Research article

Keywords: Disparities, quality of life, patient reported outcomes

Posted Date: July 22nd, 2019

DOl: https://doi.org/10.21203/rs.2.11758/v1

License: (1) (i) This work is licensed under a Creative Commons Attribution 4.0 International License.

Read Full License

Version of Record: A version of this preprint was published at Journal of Clinical Oncology on October 20th, 2018. See the published version at https://doi.org/10.1200/JC0.2018.36.30_suppl.65. 


\section{Abstract}

Background $\mathrm{A}$ better understanding of social determinants of health and psychosocial and economic sequelae of cancer for an ethnoculturally diverse population is needed. A Patient-Reported Outcomes Quality of Life (PROQOL) instrument has been developed to capture less commonly discussed patient concerns. We sought to adapt this instrument and pilot it in underserved, ethnic minority cancer patients at Maricopa Integrated Health System (MIHS) in Phoenix, AZ. Methods Two focus groups (FG) were conducted to understand patient perspectives of an adapted Spanish version of PROQOL. A revised version based on FG data was then piloted among patients on active cancer treatment. Patients also completed a Linear Analog Scale Assessment (from 1-10) for quality of life (QOL) and its domains. Results All participants in both FG (11/12 female) were Hispanic, with a median age of 53 years. Participants agreed that the domains covered in the PROQOL represented the most important psychosocial needs in their cancer continuum. No difficulty in understanding the individual domains or questions for delving deeper into each domain was noted. Median age of 34 pilot study participants (74\% female, $79 \%$ Hispanic) was 48 years. 28/34 completed the Spanish version. The most common concerns were 'Money' (32\%) and 'Cancer Diagnosis, Treatment and Survivorship' (32\%). Median overall QOL was 8 (range 3-10). Conclusions PROQOL is feasible for systematic capture of patient concerns in underserved, ethnic minority cancer patients. A better understanding of these concerns can help design interventions to improve psychosocial outcomes for the vulnerable groups and decrease disparities in care delivery.

\section{Background}

With the Commission on Cancer's requirement for developing a process to incorporate the screening of distress into standard oncological practice, healthcare systems are now routinely administering patientreported outcome measures (PROMs) to detect distress and to evaluate how quality of care affects cancer outcomes.[1] However, most PROMs have been developed and validated in populations with very low proportions of groups shown to be especially vulnerable to distress, like racial/ ethnic minorities or those with very low socioeconomic status. Previous studies have shown higher risks for adverse psychosocial outcomes in cancer survivors who face additional social stressors as poverty, low level of formal education, language barriers, and/or belonging to an ethnic or cultural minority.[2]

As has been suggested by some cross-cultural studies, the profile for psychosocial stressors may be different in the underserved populations with diverse distributions of socioeconomic variables.[3, 4] Furthermore, while there is information available about potential barriers to access that minority and underserved populations encounter, not much is known about which challenges are of greatest concern to the patient.[5, 6] Aziz and Rowland have identified absence of the right measurement systems and appropriate questions to capture the ethnic and cultural differences in the experiences of cancer care as one of the major problems in addressing disparities in the delivery of high-quality cancer care to medically underserved populations.[7] 
The Beacon Patient-Reported Outcomes Quality of Life (PROQOL) instrument was initially developed for patients with diabetes to systematically report their quality of life at the health care visit.[8] Recently, it has been modified and piloted with a predominantly Caucasian population in a Hematology and Medical Oncology practice to better understand treatment toxicity, emotional duress from the diagnosis and barriers to improved care.[9] The instrument provides patients an opportunity to report concerns on less commonly discussed issues that may impact their health management, such as social factors and personal relationships.

The goal of this study was to address the measurement and knowledge gaps in assessing distress among underserved and ethnic minorities. We used a mixed methods (qualitative and quantitative) approach to adapt and pilot the PROQOL instrument at Maricopa Integrated Health System (MIHS) and assess its feasibility for capturing the patterns and predictors of psychosocial stressors among an underserved, economically disadvantaged and ethnic minority population.

\section{Methods}

The study was approved by the IRBs at MIHS and Mayo Clinic and all patients provided informed consent in Spanish or English. Phase I of the study consisted of two focus groups (FGs) to understand patient perspectives of an adapted Spanish version of PROQOL, using grounded theory approach. In Phase II, the PROQOL was piloted among patients receiving active cancer treatment.

Focus group Participant Recruitment and Data Collection

All patients $>18$ years of age, English or Spanish speaking, undergoing active treatment for cancer at MIHS were approached in person and invited to participate while waiting to see their treating physician. A FG guide was developed by the study investigators based on clinical experience and relevant literature. The guide included open-ended questions to assess patients' understanding of PROQOL, their perceptions about its feasibility, and questions included in it to address their concerns and/or barriers to completing the instrument.

Refreshments were provided during the meeting held at the clinic and participants were given gift vouchers at the completion of the FGs. Both FGs were moderated by a clinical psychologist (PhD) at MIHS who was not a part of the study team and was assigned by MIHS to moderate the focus groups without any bias since not related to the study participants. Each FG lasted for approximately 60 minutes and field notes taken by NK and GR as well as transcribed audio recording of the session were used for the analysis.

\section{Pilot Phase Patients}

Inclusion criteria for the pilot phase were the same as for the FGs. A convenience sampling approach was used, and patients who were referred by their treating physician were approached to participate in the study between November 2017 and January 2018. Demographic and clinical data, including age, sex, 
race/ethnicity and disease characteristics, were obtained from the medical record. Median income based on residence zip code was derived from Census data and used as a proxy for patient socioeconomic status.[10]

PROQOL

The PROQOL instrument system has been developed to function as a self-reported tablet-based questionnaire that can assess symptoms/quality of life (QOL). Patient responses provide treating physicians and nurses with information regarding bothersome symptoms and/or psychosocial problems that may not be addressed normally.[8]

When completing the PROQOL on a tablet in the waiting room, patients select their single biggest concern from various categories such as: Personal Relationships, Emotional Health, Physical Health, Cancer Diagnosis and Treatment, Money and Care Planning. Patients also complete a 10-item Linear Analog Scale Assessment (LASA) to assess global QOL, and physical, emotional and social well-beings. LASA is a validated measure for QOL, where higher scores indicate better QOL.[11].[12]

\section{Analysis}

The main aim of the focus groups was to understand the participants' viewpoint about the PROQOL instruments, including its ease of understanding, feasibility and usefulness. Using standard qualitative inductive approaches, we also analyzed the qualitative data to understand other potential psychosocial concerns. We used open and axial coding to label ideas, combine them and define categories based on overlying themes in the data.

Descriptive statistics were used to describe sociodemographic and clinical characteristics of the cohort of patients enrolled in the pilot phase of the study. A univariate analysis (Kruskal Wallis test for continuous and Chi-Square test for categorical variables) was conducted to examine the association between the most commonly identified concerns and baseline sociodemographic characteristics.

\section{Results}

Baseline characteristics

A total of 31 subjects were consented for both FGs and a total of 12 attended the meeting. All participants in both FGs were Hispanics and most (11/12) were women. The median age was 53 (39-62) years. Median household income was $\$ 47,772$ (range 27,506-60,907).

Out of the fifty patients approached for the pilot study, two were found to be ineligible and 34 agreed to participate. Median age of participants was 48 (range 40-69) years; two thirds were women, $79 \%$ were Hispanic and the median household income was $\$ 42,518$ (range 24,454-101,546). Fifty three percent were married. Cancer diagnosis included solid tumor in $80 \%$ and hematological malignancy in $20 \%$ patients. (Table 1) Median time since diagnosis was 0.8 years (range 0.06 to 10.9 years). Eighty two 
percent completed the Spanish version of PROQOL. Median time to complete the instrument was 7 (range 2-18) minutes.

Focus group findings

FG participants agreed that the domains included in the PROQOL represented the most important psychosocial needs in their cancer continuum. They reported no difficulty in understanding the questions/ concerns in individual domains and did not see a need to add any other questions. One participant reported having experienced all of those concerns listed under the domain of emotional health:

"All these concerns, I have felt all of them at some point or other."(FG 1 participant \#4)

When asked about the usefulness of the PROQOL in clinic, the participants agreed that this would be a useful tool to help discuss less commonly talked about concerns.

"I think it is a good idea... I hope the doctors do take these things into consideration, and do bring them up." ( FG2 participant \#2)

Main themes around psychosocial aspects of cancer care were improving communication with providers and others, and the emotional impact of getting a cancer diagnosis. One participant noted:

"when one knows that, one gets very emotional and starts crying, and it's really hard to bring up concerns with the doctor because you're just crying, and.. you just waste time." (FG2 participant \# 3)

Participants reflected on the financial strain and increased vulnerability due to cancer.

"I understand that because all that, with the financial situation, it all piles up and you still feel the illness inside, and it leads to the depression." (FG1 participant \#6)

"I stopped as well, stopped going to a specialist, because every visit is \$100." (FG1 participant \#5)

"So, for myself, I was diagnosed with leukemia, and I needed a bone marrow transplant, but I couldn't afford it and for four years I was waiting, and finally found a research study in Maryland where I could have that done for free.. and I had to sacrifice a lot, like leaving my family for 8 months, being by myself." (FG2 participant \#5)

"once you have cancer the emotional damage and vulnerability is there. It's hard to go back." ( FG2 participant \#1)

Participants also reported a high degree of satisfaction after the FG meeting since it gave them an opportunity to engage with each other by sharing their relevant experiences, much like a support group environment. 
"...because sometimes you feel alone, and like the worst has hit you, then you hear about people that do have it a lot worse than you.. and it kind of makes you feel like you're not the only one."(FG1 participant \#3)

Concerns/ QOL assessed by PROQOL in pilot phase participants

Figure 1 shows results from the PROQOL instrument question, "What is your single biggest concern?". The three most common concerns endorsed by the pilot phase participants were 'Money' (32\%) and 'Cancer Diagnosis, Treatment and Survivorship' (32\%) and 'Physical Health' (15\%). The majority of participants who endorsed "money" as a concern reported difficulty with medical bills, non-medical costs, living expenses etc. For most participants who endorsed Cancer Diagnosis, Treatment and Survivorship concerns, it was treatment plans, including chemotherapy and use of complementary therapies that caused greatest concern. There was no difference in the distribution of concerns based on age, sex, marital status, type of cancer or ethnicity.

Median overall QOL was 8 (range 3-10), suggesting relatively high QOL. Figure 2 shows the mean LASA scores in the different domains for the FG participants.

\section{Discussion}

The increasing diversity of the U.S. population has drawn attention to the need for developing culturally relevant methods in order to integrate the physiologic, psychosocial, and economic sequelae of cancer and its treatment into routine healthcare delivery. For ethnoculturally diverse and medically underserved populations, a better understanding of their challenges is needed to design interventions to address their less commonly discussed concerns and decrease disparities in care delivery. In this mixed methods study, we were able to show that PROQOL is feasible for systematic capture of patient concerns in a medically underserved patient population with ethnic minority cancer patients. Desire for more information about the cancer diagnosis and treatment options, financial concerns and concerns regarding physical health emerged to be the most important concerns for this population. Difficulty in accrual due to patients' beliefs and attitudes is a well-known barrier for conducting such studies in an under-represented population.[13, 14] Surprisingly, our use of study staff trained to work with minority populations, communication about the study in a culturally appropriate, respectful, and easy to understand way and use of Spanish study instruments helped in accruing more than $50 \%$ of approached patients and retaining them on the study with not too much difficulty. Though seven minutes is a long time to complete the instrument, this was mostly due to the participants' discomfort in handling the tablet. A paper version was available for those not able to do it on the tablet, but all participants ended up completing the tablet version. Also, this was completed in the waiting room prior to the physician appointment, so we do feel it would be feasible without interfering much with appointment times and workflow.

Increased psychosocial distress and poor quality of life (both overall and in some specific dimensions only) have been reported extensively in cancer survivors.[15-17] Opportunities to screen for and improve 
psychosocial concerns exist throughout the spectrum of cancer care from diagnosis to end-of-life care. As the proportion of minorities in the US population continues to grow, it creates a greater imperative to understand the racial/ethnic disparities in the spectrum of psychosocial concerns that may impact cancer outcomes eventually.

Studies that have reported on quality of life or psychological problems in minorities have been done using standardized instruments to capture the outcomes. Bowen et al have shown poorer physical functioning in 'Blacks' and 'other ethnic groups' as compared to Whites.[18] Somewhat conflicting results were reported in a qualitative study of breast cancer survivors in a multi-ethnic group which revealed that the overall HRQOL was not significantly different between various ethnicities. However, the authors noted that economic barriers were particularly important for African American and Hispanic survivors.[19] In another study on psychological distress during cancer survivorship, being African American was reported to be related to fewer cancer-related health worries.[20] Although income and socioeconomic status is closely intercalated in the web of racial/ethnic disparities, the literature on impact of income on psychosocial concerns is limited too. A study that evaluated the income disparities in quality of life among cancer survivors showed poorer quality of life in low income groups.[21] Lower household income has been related to both the treatment related financial hardship as well as adverse psychological outcomes.[22,23] Evidence suggests that the biopsychosocial burden of cancer may be particularly high for certain ethnic groups. Latinas were more likely to report poor mental, physical and social QOL when compared with non-Latina Whites and Blacks, as well as lower perceived social support, high levels of uncertainty and higher levels of depression.[19,24] Hispanic cancer survivors had the highest level of unmet supportive care needs (informational, practical, supportive and spiritual) when compared with African Americans and Whites.[25] Data from our pilot phase also showed the difference in spectrum of concerns identified by PROQOL when administered to a predominantly Caucasian population at Mayo Clinic vs. the minority population at MIHS. [9] While 'Cancer Diagnosis, Treatment and Survivorship' was one of the two top concerns for both cohorts, 'Money' emerged as an equally important concern in the minority population as opposed to 'Physical Health' in Caucasian population.

The PROQOL also provides a platform to develop suggested actions as a template that local health care teams can adapt to reflect local community resources and their specific team's structure or method of operation. System level factors may need policy solutions targeted towards minority populations, but other potential patient-centered interventions to help address these concerns could range from increased psychosocial and tangible support with involvement of medical staff, community resources, patient navigators, and patient support groups.

There are a few limitations to our study. Because we did not have the individual level sociodemographic data, we used census data as a proxy for income. The convenience sampling approach for the focus groups may have resulted in selection of a subset of patients with specific diagnoses and may not capture the extent of diversity of perceptions. However, our pilot phase patients were consecutive patients coming to the cancer center clinic and did represent a more heterogeneous group in terms of the disease spectrum. A small sample size for the pilot study limited the assessment of associations between the 
concerns and sociodemographic characteristics. Finally, while the use of IT made the process more streamlined, it also raised obstacles in trying to work across the firewalls of different institutions and technical difficulty reported by some subjects in answering the questions on a tablet.

Despite the limitations, our exploratory study with a high proportion of underserved population addresses gaps in the literature on health disparities and health care delivery. It provides valuable information about the feasibility and acceptability of trying to integrate PROs into clinical practice while taking care of a very vulnerable group of patients. Additional research, including a larger multi-center study with the PROQOL is needed along with development of care pathways that allow for greater engagement of the entire clinical team, staff, and community resources to address the concerns raised by the patient. This would also require an understanding of the providers' perspectives around integration of such an instrument in clinical workflow and its impact on workflow and clinical burden.

\section{Conclusion}

The PROQOL provides a reasonable option to address barriers in delivery of psychosocial health services and leverage the case management framework to address physical and psychosocial consequences of cancer and treatment even in a medically challenging ethnic minority and underserved population.

\section{Abbreviations}

Patient-Reported Outcomes Quality of Life (PROQOL); focus group (FG); quality of life (QOL); patientreported outcome measures (PROMs); Maricopa Integrated Health System (MIHS)

\section{Declarations}

\section{- Ethics approval and consent to participate}

The study was approved by the IRBs at MIHS and Mayo Clinic and all patients provided informed consent in Spanish or English.

\section{- Consent for publication}

NA

\section{- Availability of data and material}

The data that support the findings of this study are available from MIHS but restrictions may apply to the availability of these data, which are not publicly available. Data are however available from the authors upon reasonable request and with permission of MIHS.

\section{- Competing interests}


No conflict of interest for any authors.

\section{- Funding}

The project was funded by Mayo Clinic Investment for Extramural Grants Award (MEGA) Program

\section{- Authors' contributions}

NK, JS and JG designed the study. NK conducted the literature search and wrote the first draft of the manuscript. BF did the statistical analyses. IK, WA and GR contributed patients. All authors assisted in interpretation of the findings, offered critical review of the paper, and approved the final manuscript.

\section{- Acknowledgements}

NA

\section{References}

1. Cancer Program Standards 2012 (version 1.2): Ensuring Patient-Centered Care Available from: [http://facs.org/cancer/coc/programstandards2012.html.]. Accessed December 21, 2018 2. Maly RC, Umezawa Y, Ratliff CT, Leake B: Racial/ethnic group differences in treatment decision-making and treatment received among older breast carcinoma patients. Cancer 2006, 106(4):957-965. 3. Burgess C, Cornelius V, Love S, Graham J, Richards M, Ramirez A: Depression and anxiety in women with early breast cancer: five year observational cohort study. BMJ 2005, 330(7493):702. 4. Napoles-Springer AM, Ortiz C, O'Brien H, Diaz-Mendez M: Developing a culturally competent peer support intervention for Spanishspeaking Latinas with breast cancer. J Immigr Minor Health 2009, 11(4):268-280. 5. Shavers VL, Brown ML: Racial and Ethnic Disparities in the Receipt of Cancer Treatment. Journal of the National Cancer Institute 2002, 94(5):334-357. 6. Disparities in Cancer Care. Journal of Oncology Practice 2006, 2(5):234239. 7. Aziz NM, Rowland JH: Cancer Survivorship Research Among Ethnic Minority and Medically Underserved Groups. Oncology nursing forum 2002, 29(5):789-801. 8. Ridgeway JL, Beebe TJ, Chute CG, Eton DT, Hart LA, Frost MH, Jensen D, Montori VM, Smith JG, Smith SA et al: A brief Patient-Reported Outcomes Quality of Life (PROQOL) instrument to improve patient care. PLoS Med 2013, 10(11):e1001548. 9. Warsame RM, Thompson CA, Hubbard JM, Fonder AL, Hobbs MA, Hwa L, Kourelis T, Gonsalves WI, Croghan K, Fruth B et al: What are patients' biggest concerns? A patient reported outcome case-management system. Journal of Clinical Oncology 2017, 35(15_suppl):6572-6572. 10. 2013-2017 American Community Survey 5-Year Estimates. Available from:

[https://factfinder.census.gov/faces/nav/jsf/pages/community_facts.xhtml]. Accessed December 13, 2018 11. Singh JA, Satele D, Pattabasavaiah S, Buckner JC, Sloan JA: Normative data and clinically significant effect sizes for single-item numerical linear analogue self-assessment (LASA) scales. Health Qual Life Outcomes 2014, 12:187. 12. Locke DEC, Decker PA, Sloan JA, Brown PD, Malec JF, Clark MM, Rummans TA, Ballman KV, Schaefer PL, Buckner JC: Validation of Single-ltem Linear Analog Scale Assessment of Quality of Life in Neuro-Oncology Patients. Journal of pain and symptom management 
2007, 34(6):628-638. 13. Howerton MW, Gibbons MC, Baffi CR, Gary TL, Lai GY, Bolen S, Tilburt J, Tanpitukpongse TP, Wilson RF, Powe NR et al: Provider roles in the recruitment of underrepresented populations to cancer clinical trials. Cancer 2007, 109(3):465-476. 14. Ford JG, Howerton MW, Lai GY, Gary TL, Bolen S, Gibbons MC, Tilburt J, Baffi C, Tanpitukpongse TP, Wilson RF et al: Barriers to recruiting underrepresented populations to cancer clinical trials: A systematic review. Cancer 2008, 112(2):228-242. 15. Dabrowski M, Boucher K, Ward JH, Lovell MM, Sandre A, Bloch J, Carlquist L, Porter M, Norman L, Buys SS: Clinical Experience with the NCCN Distress Thermometer in Breast Cancer Patients. Journal of the National Comprehensive Cancer Network 2007, 5(1):104-111. 16. Donovan KA, Jacobsen PB: Progress in the Implementation of NCCN Guidelines for Distress Management by Member Institutions. Journal of the National Comprehensive Cancer Network 2013, 11(2):223-226. 17. Ganz PA, Desmond KA, Leedham B, Rowland JH, Meyerowitz BE, Belin TR: Quality of Life in Long-Term, Disease-Free Survivors of Breast Cancer: a Follow-up Study. Journal of the National Cancer Institute 2002, 94(1):39-49. 18. Bowen D, Alfano C, McGregor B, Kuniyuki A, Bernstein L, Meeske K, Baumgartner K, Fetherolf J, Reeve B, Smith A et al: Possible socioeconomic and ethnic disparities in quality of life in a cohort of breast cancer survivors. Breast Cancer Res Treat 2007, 106(1):85-95. 19. Ashing-Giwa KT, Padilla G, Tejero J, Kraemer J, Wright K, Coscarelli A, Clayton S, Williams I, Hills D: Understanding the breast cancer experience of women: A qualitative study of African American, Asian American, Latina and Caucasian cancer survivors. Psychooncology 2004, 13(6):408-428. 20. Deimling GT, Bowman KF, Sterns S, Wagner LJ, Kahana B: Cancer-related health worries and psychological distress among older adult, long-term cancer survivors. Psychooncology 2006, 15(4):306-320. 21. Short PF, Mallonee EL: Income disparities in the quality of life of cancer survivors. Medical care 2006, 44(1):16-23. 22. Sun CL, Francisco L, Baker KS, Weisdorf DJ, Forman SJ, Bhatia S: Adverse psychological outcomes in long-term survivors of hematopoietic cell transplantation: a report from the Bone Marrow Transplant Survivor Study (BMTSS). Blood 2011, 118(17):4723-4731. 23. Shankaran V, Jolly S, Blough D, Ramsey SD: Risk factors for financial hardship in patients receiving adjuvant chemotherapy for colon cancer: a population-based exploratory analysis. $\mathrm{J}$ Clin Oncol 2012, 30(14):1608-1614. 24. Yanez B, Thompson EH, Stanton AL: Quality of life among Latina breast cancer patients: a systematic review of the literature. J Cancer Surviv 2011, 5(2):191-207. 25. Moadel AB, Shah C, Wylie-Rosett J, Harris MS, Patel SR, Hall CB, Sparano JA: Randomized controlled trial of yoga among a multiethnic sample of breast cancer patients: effects on quality of life. $\mathrm{J}$ Clin Oncol 2007, 25(28):4387-4395.

\section{Figure Legends}

Figure 1: Biggest concern identified on PROQOL

Figure 2: Mean Linear Analog Scale Assessment (LASA) Scores for pilot study participants

\section{Table}

Table 1: Baseline characteristics 
Characteristics

Focus group participants $(n=12)$

Median age (years)

Gender (\% female)

Race/ Ethnicity (\%)

- Hispanic White 100

- Non-Hispanic White

0

0

91

9

- Heme Malignancy

Marital Status (\%)

- Married

67

0

33

Median household income

53

91

- Single

- $\quad$ Other
80

74

Pilot study participants $(n=34)$

48

10

10

79

21

53

32

5

$\$ 42,518$

\section{Figures}


Figure 1: Biggest concern identified on $P R O Q O L$

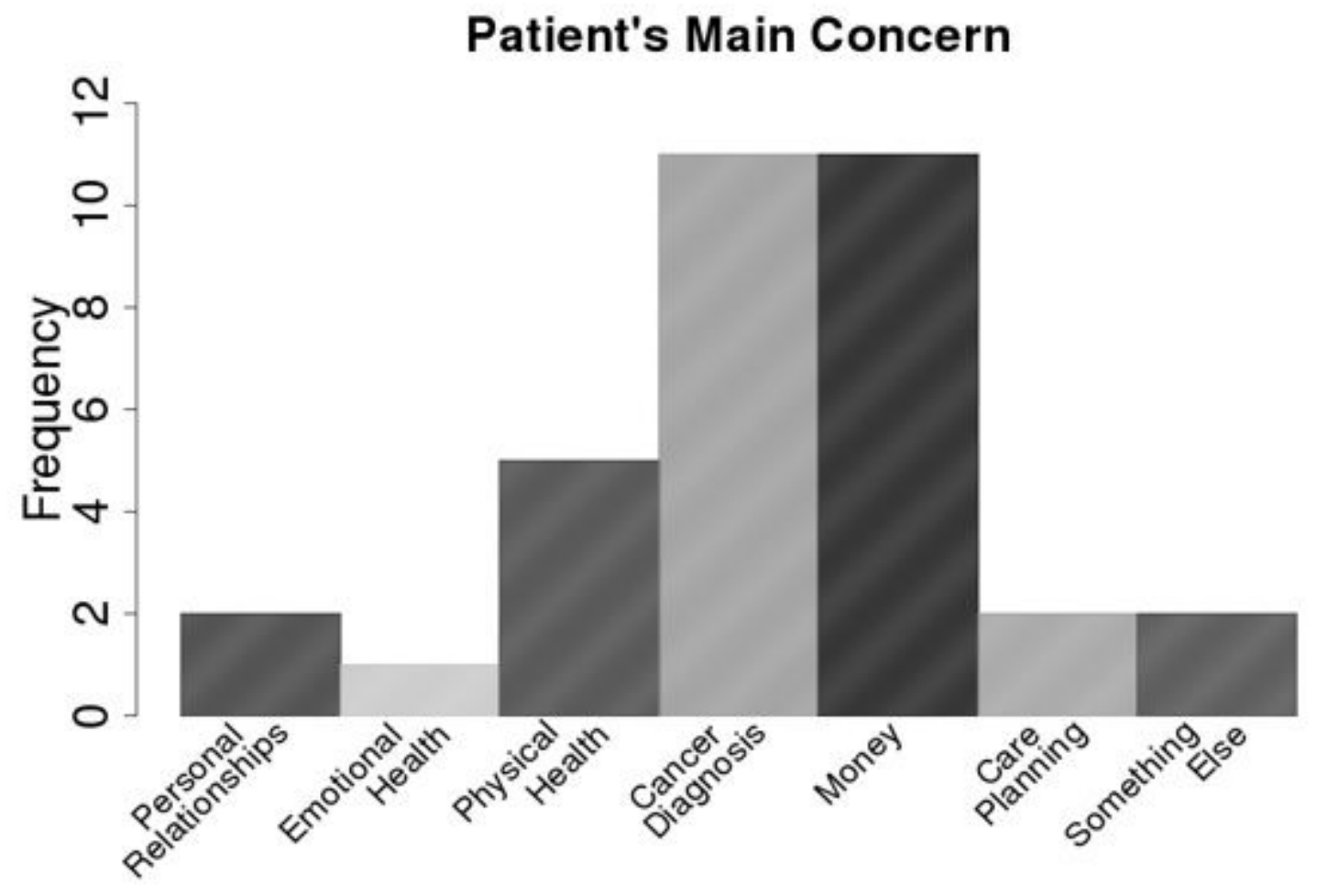

Figure 1

Biggest concern identified on PROQOL 
Figure 2: Mean Linear Analog Scale Assessment (LASA) Scores for pilot study participants

\section{Mean Lasa Scores By Arm}

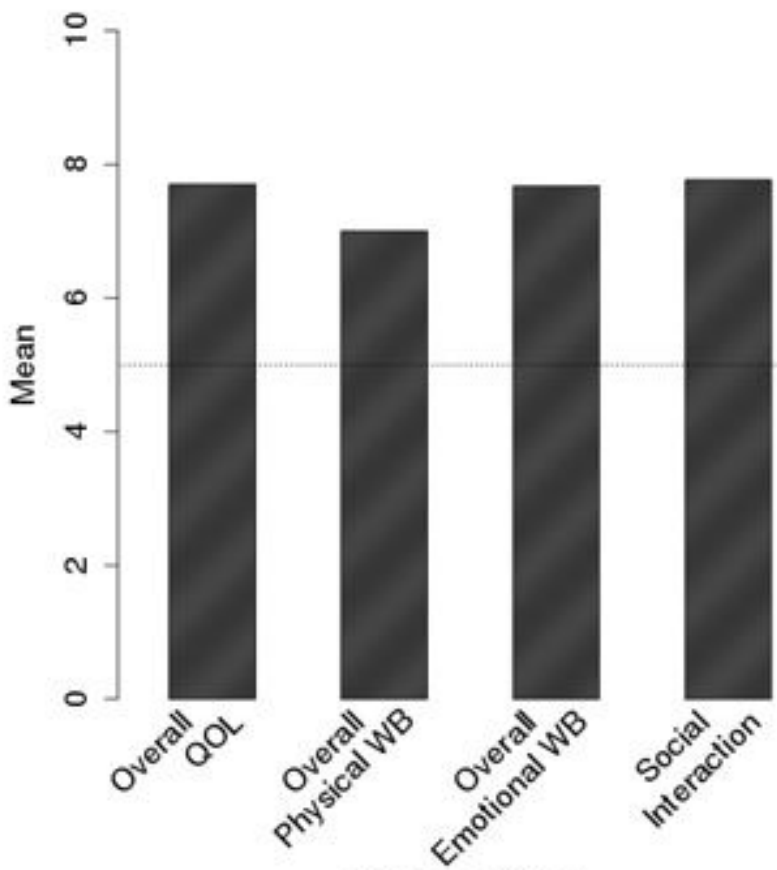

-Higher is Better

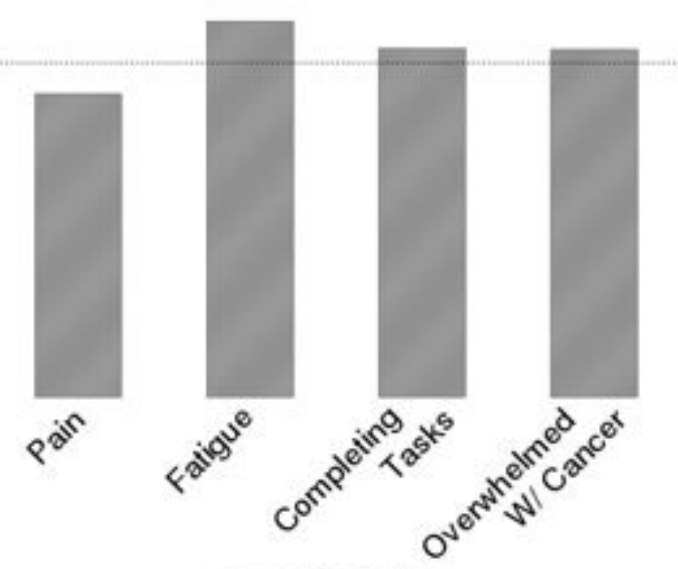

-Lower is Better

\section{Figure 2}

Mean Linear Analog Scale Assessment (LASA) Scores for pilot study participants

\section{Supplementary Files}

This is a list of supplementary files associated with this preprint. Click to download.

- ISSMCOREQChecklistnk.pdf 